\title{
Worldwide mobile wallet: a futuristic cashless system
}

\author{
Qorina Mailil Husna Mumtaza ${ }^{\mathrm{a}, 1, *}$, Shada Intishar Nabillah, ${ }^{\mathrm{a}, 2}$, Sholikhatul \\ Amaliya $^{\mathrm{a}, 3}$,Yuveta Rosabella ${ }^{\mathrm{a}, 4}$, Jehad A.H Hammad,. \\ ${ }^{a}$ Department of Electrical Engineering, Universitas Negeri Malang, Malang, Indonesia \\ ${ }^{\mathrm{b}}$ Department of Computer Information Systems, Al-Quds Open University (QOU), Palestine \\ ${ }^{1}$ qorinamumtaza@gmail.com; ${ }^{2}$ shadaintishar@gmail.com; ${ }^{3}$ sholikhatul.lia@gmail.com; ${ }^{4}$ yu vetarosabella23@gmail.com; ${ }^{5}$ jhammad@qou.edu \\ * corresponding author
}

\section{ARTICLE INFO \\ Article history \\ Received July 24, 2020 \\ Revised August 10, 2020 \\ Accepted August 30, 2020 \\ Keywords \\ Futuristic \\ Cashless system \\ Mobile wallet}

ABSTRACT

The proliferation of the internet led to the emergence of new technology. Nowadays, many activities can be done online, such a payment. After Mobile Banking was introduced publicly, a new method for payment called mobile wallet that uses mobile application as a device appears. The ease caused by this technology should be the main reason for implementing this system on to daily basis. Nevertheless, in various countries, the usage rate of the cashless system remains uneven, caused by many factors, both from internal factors such as security risks and external factors such as the policies of the countries involved. This paper will evaluate worldwide online payment systems to determine the impact of online payment systems usage, both positive and negative. This paper will evaluate the factors that affect the introduction of m-payment. Several measurements, such as country with the highest and lowest cashless payment usage and the reasons were taken for this research so that the objectives to improve the system and increase the usage rate can be achieved.

\section{Introduction}

The Cashless system is the latest breakthrough for payments method, where the use of physical money is no longer used and replaced by virtual cash. There are many types of cashless systems such as the use of mobile banking, credit cards, online wallets, etc. that can be used to replace the use of physical money. There are several conveniences from cashless system which hopefully can help the economic sector handle some of their problems, such as the ease of conducting transaction on daily basis, reduce the burden of the cost of printing currency, tracks the movement of money easily, and it also expected to reduce the amount of corruption because the entire digital financial process is carried out transparently, where money that has been used can be traced back more easily than when using physical money, and lower transaction cost [1]-[4].

Abrazhevich [5] explains that credit cards are the most dominant cashless payment method used on an international scale. In United States, 95\% of all e-commerce transactions are performed using credit cards. However, credit card ownership has several restrictions that cannot be met by everyone, this is different from using a mobile wallet. Currently, mobile wallet is becoming a trend as a payment method only in various countries and its use is limited to small payments for purchasing mobile games, movie tickets, and content online. Mobile wallet is a payment method using a smartphone application that has digital money for transaction, digital cards, digital receipts, and digital coupons. From the definition, the features offered by mobile wallets are more advantageous than other cashless system. Some mobile wallets also provide physical card for cash withdrawal. 
Nonetheless, with various facilities provided by mobile wallets, its use is still uneven and only a few countries are interested in adopting this payment. There are several negative impacts such as the possibility of turning citizen into criminals such as commit IT crime, and the difficulties to organized criminal and networks who take advantage of the regulations and legal infrastructures that governments put in place decrease the adopting value of mobile wallet systems [6], [7]. Ayoola [8] researched the effect of the cashless system in Nigeria, and as a result, one of the goals of the cashless system, which was to reduce corruption, was not successful. Besides, Nigeria has only $29 \%$ for rate of smartphone and mobile penetration [9]. Cisar [10] states that a cashless system is considered to reduce only petty corruption and cashless policy actually just shifting corruption from one group of society to another and not really reducing it. Complexity for the use of cashless payment and security concerns also contributed to the low adoption of this payment method.

Study in India [1], [11]-[13] given reasons why mobile wallets are difficult to be adopted there. Such a lack of access to banking where physical money will be used continuously because most people in India are not connected with the banks, and only highly educated people were interested in cashless transactions. It is only $35 \%$ of India's population connected with the internet, Illiteracy, where around $30 \%$ of the people do not know how to read, write, use computer and internet, lack of infrastructure, and extra charges for online transactions.

The cause of the low rate of adoption of mobile wallets in various countries is certainly different. Factors in India and Nigeria cannot be used as benchmarks for the level of implementation of worldwide mobile wallets and cannot be used as a reference for how to increase the use of mobile wallet as a new payment method. Therefore, analysis of various countries is needed to determine most problems so that the solutions can be produced.

\section{Method}

This study is based on secondary sources of information. This study's target population consisted of adult worldwide mobile phone users who have downloaded a mobile payment application. The data were collected through online statistical institutions in all parts of the world. We also use comparative analysis to compare the differences and similarities of the facts.

\section{Result and Discussion}

The use of cashless payment currently continues to set its inexorable rise and become the secondbest common payment method after debit cards (Fig. 1). The sixth annual Mobile Payments and Fraud Survey conducted in 2018 by Kount found that $37 \%$ of the merchants that participated already supported mobile payments at the Point of Sale and $31.4 \%$ planned to add to this feature or increase it.

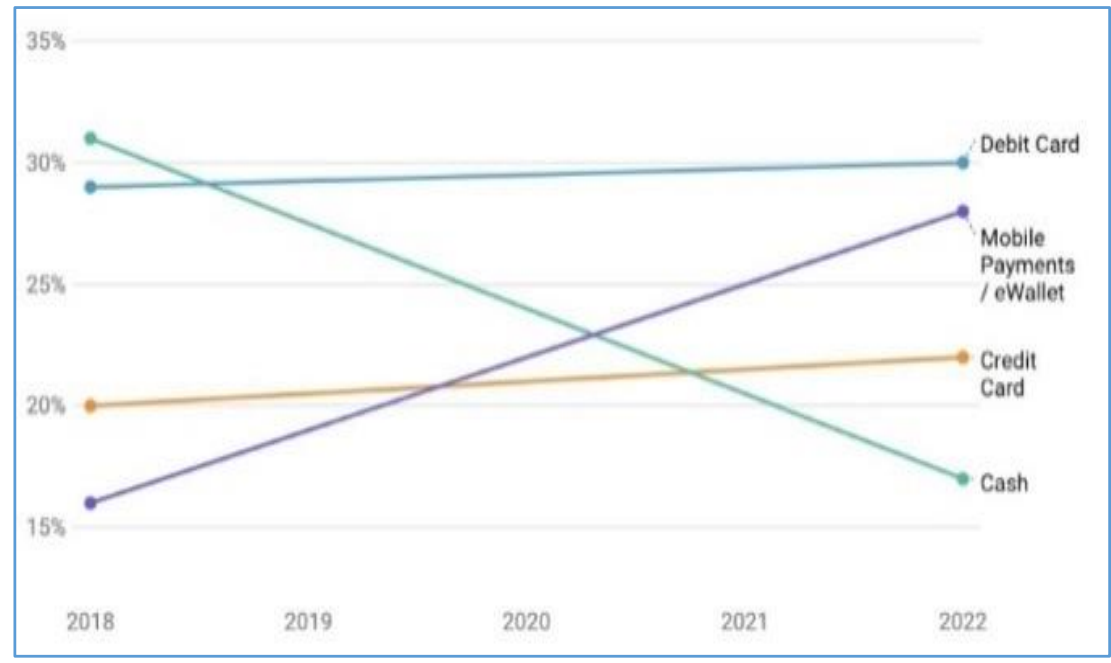

Fig. 1. Global Share of Point of Sale Payment Methods / Data Source: Worldpay's 2018 Global Payment Report 
Table 1. Worldwide Biggest Mobile Payment Income

\begin{tabular}{ccc}
\hline Company & Active Users & Latest Figures From \\
\hline WeChat & 1 billion+ & Tencent (Jan 2019) \\
Alipay & 1 billion + & Alipay (Jan 2019) \\
PayPal & 250 million & Paypal (Sep 2018) \\
Apple Pay & 383 million & Loup Ventures; QZ (Feb 2019) \\
Amazon Pay & 50 million & Evercore ISI, Investopedia (May 2018) \\
Samsung Pay & 1 billion+ & Statista (Aug 2017) \\
Google Pay & 24 million & Statista (Aug 2017) \\
\hline
\end{tabular}

At present, Asia Pacific dominates the global absorption of e-wallets and POS payments (Fig. 2). Southeast Asia is one of the fastest-growing technological regions in the world. China is one of the biggest supporters of e-wallet and research from eMarketer shows that more than $45.2 \%$ of the Chinese population already uses mobile payments (Table 1). China dominates technology companies in the cellular payment industry with a large population also has the highest percentage of respondents who say they prefer to use a mobile payment platform [14]. China also has complex connections with Hong Kong and Taiwan, but unfortunately, Hong Kong and Taiwan are slower than China to adopt mobile payments.

Singapore as part of the Asia Pacific is leading mobile payments following China because it has $85 \%$ smartphone supplies, high average salary, and a strong consumption culture [15]. Although the population is smaller than in Indonesia, Singapore's cellular payment market is worth more than $\$ 1$ billion. Although Singapore has led the growth in the use of a cashless system in Southeast Asia, a survey shows that Singaporeans still prefer to use cash and cards as the main transaction tool. While driving the transition towards digital payments, the Monetary Authority has released e-payment guidelines that are aimed at protecting consumers and driving wider adoption of such platforms in the country. The balance between innovation and regulation has become a focus area to protect consumers, which is more important after major data violations. The big obstacle in the development of a cashless payment culture in Southeast Asia is not caused by a lack of smartphone supply but is caused by many people who still do not have a bank account. Only about $27 \%$ of people in all regions have bank accounts.

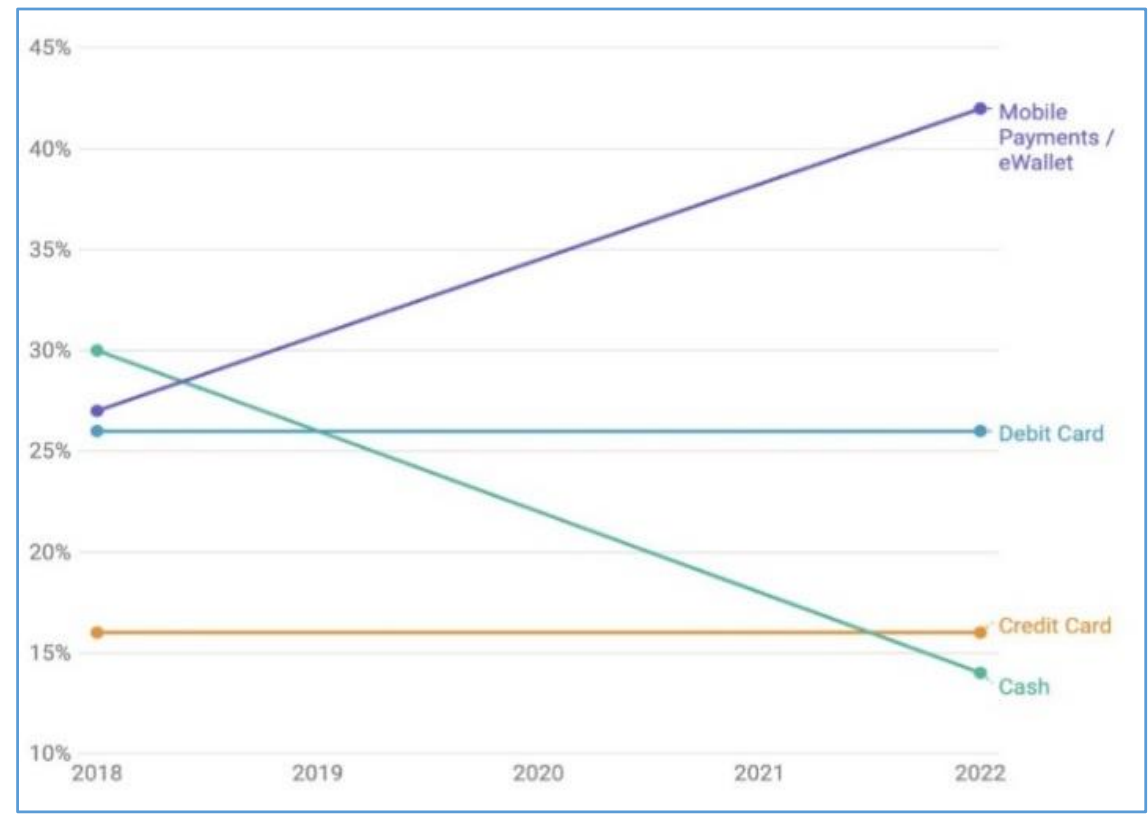

Fig. 2. Chart Asia Pacific Point of Sale Payment Methods Forecast 2018 - 2022 / Data Source: Worldpay's 2018 Global Payment Report

In contrast to the US, which has one of the highest smartphone adaptation rates in the world, it is lagging in mobile payments. Based on research from Simon-Kucher \& Partners [16], almost 90\% of 
US respondents would rather pay with cash, credit cards, or debit cards rather than cellular alternatives because they are not confident in using them and feel that they do not understand technology enough. The only cashless payment they used to use was the Starbucks application and Apple Pay. This shows that the US Cellular Payment Market is 35 times smaller than the world leader, China.

The use of global payments in North America is expected to increase from $16 \%$ in 2018 to $28 \%$ in 2022. This is still far below the level of global adoption, but this is a good step forward. Meanwhile, throughout Europe, Sweden is the largest cashless country in Europe with 77\% of the population already using mobile banking or cellular payments, followed by the UK and France [17].

The survey shows that the increase in the use of non-cash payments in the European region has increased quite rapidly. If we explore earlier, Europe is a little slower to start using payments without cellular cash. In the shadow of the Facebook scandal, Sweden is afraid of data privacy risks and is considering new regulations to protect consumer privacy in a cashless era. The Swedish central bank has already urged lawmakers to protect cash payments, but a survey confirmed that Sweden wants to keep cash as an option for transactions [18].

Unknown to many, Norway might be the first cashless country ever with only less than $10 \%$ of the population uses cash [9]. With this fantastic number of users, Norway is one of the leaders in Europe who has used cashless payments. While Europeans show mobile payments are growing significantly in their communities, they also express concern about privacy and security is still prevalent. This shows that infrastructure-related improvements are needed to create a culture of payment without cash.

Africa has been opened by the smartphone market which is incorporating devices and allowing access to e-commerce applications. Throughout 2018, Africa has spread technological innovations to overcome the challenges of Africa in mobile payments [19]. Given the fact that technological challenges have arisen throughout the years, Africa faces the risk of financial crime that underestimates the benefits of Africa's economic growth. Several countries in Africa such as Zimbabwe and Kenya also experienced nervous periods through technical disturbances from the dominant market, echoing concerns from events in other regions about dependence on technology [20]. Even with cybersecurity risks, Zimbabwe has led to the use of cellular payments since 2017. In 2017, cellular payments grew substantially because of cash shortages.

From the results of the analysis of the impact on the adoption of mobile wallet, advantages and reasons for the difficulty of implementing this system as a payment method, and also the level of adoption of this system worldwide, we propose solutions that must be done to develop mobile wallets so that the use of this system will increase.

The system may enhance user trust. Building user trust is not just by doing massive promotions, giving gift coupons, or forcing their use in any way. Forced use usually occurs, for example, only providing payment methods with a mobile wallet and does not accept other types of payment. This only causes inconvenience to the user. Promotion or gift coupons do not have a significant impact if the problem is user distrust. Thus, mobile wallets must build public trust. The public is convinced that the use of a mobile wallet as a payment method is genuinely secure, making transactions more comfortable and convenient.

Mobile wallets improves security aspects. Concerns about using a mobile wallet are insecurity about identity theft, data misuse, and loss of money due to hacking. Hence, not only convincing users that the mobile wallet system has guaranteed security, the security aspects and policies that can support those security aspects must also be improved.

Cashless policy to regulate the use of this system so that it is not misused.Make regulations to prevent mobile wallets from being misused media, such as money laundering, fraud, duplicating accounts with different data, etc.

One of the factors causing the difficulty of mobile wallets being used as the latest payment method is that people have a problem using it, which is considered too complicated. From the analysis of users in the United States, the low usability and user satisfaction from most mobile wallets for certain circles caused the complexity. Mobile wallets should make the system easy to use 
for those who have experience in this technology and those who are accustomed to using other payment methods.

The lack of infrastructure is one reason why mobile wallets are difficult to apply in various countries. Support for implementing mobile wallets, for example, scanning machines, non-ATM top-up machines, and machines to withdraw balances because not all mobile wallets provide access to transfer balances to user accounts.

\section{Conclusion}

A mobile wallet can be the future of a cashless system because of its positive impact. However, the use of this method is still uneven, with only a few countries adopting mobile wallet as one of the payment methods in their country. After an evaluation that revealed the positive and negative impacts of the use of mobile wallets and the reasons for the difficulty of adopting this method by taking data and studies from various countries around the world, some steps can be taken to develop and expect mobile phones in the future. Wallets can be applied on a larger scale. These steps include enhancing user trust, improving security aspects, making regulations that protect the legal use of mobile wallets, reducing the complexity of mobile wallets, and adding infrastructure to support mobile wallets. According to our work, in the future, the mobile wallet system should improve the way they build their relationship with customers. Then, each country involved in the adoption of mobile wallets as one of their payment methods must develop regulations that cannot provide crevices to be misused and ensure that the system is safe to use.

\section{References}

[1] W. Akram, "Challenges and opportunities experienced in moving from cash to cashless economy," Int. J. Curr. Trends Sci. Techno, vol. 7, no. 8, pp. 20239-20243, 2018.

[2] P. Sarika and S. Vasantha, "Impact of mobile wallets on cashless transaction," Int. J. Recent Technol. Eng., vol. 7, no. 6, pp. 1164-1171, 2019.

[3] N. Ramya, D. Sivasakthi, and M. Nandhini, "Cashless transaction: Modes, advantages and disadvantages," Int. J. Appl. Res., vol. 3, no. 1, pp. 122-125, 2017.

[4] N. Fabris, "Cashless society - The future of money or a Utopia?," J. Cent. Bank. Theory Pract., vol. 8, no. 1, pp. 53-66, 2019, doi: 10.2478/jcbtp-2019-0003.

[5] D. Abrazhevich, Electronic payment systems: A user-centered perspective and interaction design. 2004.

[6] J. W. Rivera, "Potential negative effects of a cashless society: Turning citizens into criminals and other economic dangers," J. Money Laund. Control, vol. 22, no. 2, pp. 350-358, 2019, doi: 10.1108/JMLC-042018-0035.

[7] R. Apau, E. Obeng, and A. N. Darko, "An empirical evaluation of cashless systems implementation in Ghana," Int. J. Business, Econ. Manag., vol. 6, no. 3, pp. 159-173, 2019, doi: 10.18488/journal.62.2019.63.159.173.

[8] T. Ayoola, "The effect of cashless policy of government on corruption in Nigeria," Int. Rev. Manag. Bus. Res., vol. 2, no. 3, pp. 682-690, 2014.

[9] M. Humbani and M. Wiese, "A cashless society for all: Determining consumers' readiness to adopt mobile payment services," J. African Bus., vol. 19, no. 3, pp. 409-429, 2018, doi: 10.1080/15228916.2017.1396792.

[10]O. Cisar, "Strategies for using information technologies for curbing public-sector corruption: The case of the Czech Republic (CR)," Word J. Int. Linguist. Assoc., 2003.

[11]N. Kumari and J. Khanna, "Cashless payment: A behaviourial change to economic growth," Int. J. Sci. Res. Educ., vol. 5, no. 7, pp. 6701-6710, 2017, doi: 10.18535/ijsre/v5i07.03.

[12] S. Chandra and P. Mittal, "A study of cashless transaction behavior of Bank customers in district Mathura, up," Scholarly Research Journal for Humanity Science \& English Language, vol. 6, no. 26. 2018, doi: 10.21922/srjhsel.v6i26.11394. 
[13] V. Podile and P. Rajesh, "Public perception on cashless transactions in India," Asian J. Res. Bank. Financ., vol. 7, no. 7, pp. 63-77, 2017, doi: 10.5958/2249-7323.2017.00069.4.

[14] J. Clement, "Countries with the highest share of internet users preferring mobile payments as of August 2017." 2019.

[15] Worldpay, "The art and science of global payments a definitive report from worldpay.” p. 108, 2018.

[16] W. Ke, D. Chung, W. Li, and J. Furgiuele, "How behavioral science can unleash digital payments adoption," 2018.

[17] M. Jain, "Making towards a Cashless Economy: Challenges and Opportunities for India," Indian J. Appl. Res., vol. 7, no. 1, pp. 722-723, 2017.

[18] M. Savage, "The Swedes rebelling against a cashless society," Bussiness reporter. 2018.

[19] A. Bose and B. Mellado, “World Payments Report 2018.” Capgemini and BNP Paribas, 2018.

[20] gSM Association, "The mobile economy sub-Saharan Africa," GSMA Intelligence. GSM Association, London, 2017, doi: 10.5121/ijcsit.2015.7409. 